\title{
Cutaneous Protothecosis with Meningitis Due to Prototheca wickerhamii in an Immunocompetent Teenager: Case Report and Literature Review
}

\author{
Yanfei Lu (iD) 1,2,* \\ Xiaohui Zhang (iD) ${ }^{1,2, *}$ \\ Fang $\mathrm{Ni}^{1,2}$ \\ Wenying Xia (iD) ${ }^{1,2}$ \\ 'Department of Laboratory Medicine, \\ Jiangsu Province Hospital and Nanjing \\ Medical University First Affiliated \\ Hospital, Nanjing, People's Republic of \\ China; ${ }^{2}$ National Key Clinical \\ Department of Laboratory Medicine, \\ Nanjing, People's Republic of China \\ *These authors contributed equally to \\ this work
}

Correspondence: Wenying Xia Department of Laboratory Medicine, Jiangsu Province Hospital and Nanjing Medical University First Affiliated Hospital, Street No. 300, Guangzhou, 210029, People's Republic of China

Tel +8625-6830-6287

Fax +8625- 8372-4440

Email xiawenying21106891@163.com

\begin{abstract}
Human protothecosis is a rare infection caused by Prototheca spp., which are environmental achloric algae ubiquitously existing in nature. Members of the genus of Prototheca usually cause localized infection that affects the skin or wounds. Systemic infection is extremely rare and tends to occur in immunocompromised patients. Here, we report a case of cutaneous protothecosis and meningitis due to Prototheca wickerhamii in an immunocompetent teenager who obtained full-body tattoos at the time of infection. To the best of our knowledge, this is the first description of $P$. wickerhamii isolated from both skin tissue and cerebrospinal fluid. The data contained in this report will increase our understanding of this pathogen and elucidate the most optimal treatment.
\end{abstract}

Keywords: Prototheca wickerhamii, cutaneous protothecosis, meningitis, immunocompetent

\section{Introduction}

Prototheca spp. are achlorophyllous green algae that are classified in the Chlorellaceae family. They are ubiquitous and can be isolated from water, grass, trees, animals, food items, and even household garbage. ${ }^{1}$ Human protothecosis is a rare infection mainly caused by Prototheca wickerhamii and Prototheca zopfii, with the former being more common. ${ }^{2}$ The main manifestations include cutaneous infection, olecranon bursitis, and disseminated infection, among which disseminated infection is infrequent and mainly occurs in immunocompromised patients. ${ }^{3}$

Here, we report a cutaneous Protothecosis infection that progressed to meningitis due to $P$. wickerhamii in an immunocompetent teenager. Only five cases of meningitis due to $P$. wickerhamii have been reported in the literature to date, and this is the first description of $P$. wickerhamii isolated from both skin tissue and cerebrospinal fluid (CSF). Clinicians should be alert to the possibility of progression from a localized infection to aggressive infection in immunocompetent hosts. Written informed consent was provided by the patient to allow the case details to be published, and our study was approved by the Ethics Committee at Jiangsu Province Hospital.

\section{Case Report}

A 17-year-old boy presented to our dermatological department with systemic erythema erosion accompanied by itching pain and fever in October 2020. He was previously in satisfactory condition. He had a history of full-body tattoo in 
May 2019. Ten months ago, the patient developed a wheal-like rash with pruritus with no apparent predisposing cause that was followed by herpes, erosion, and ulceration. A month ago, the symptoms gradually spread to his entire body, especially the face and neck, with swelling and pain in the lip, and he developed a fever with a temperature of $38.2^{\circ} \mathrm{C}$.

He received a diagnosis of eczema and herpetic dermatitis in other hospitals, where symptomatic treatment was administered, with cephalosporin for infection, but rash control was poor. Despite oral administration of cefdinir, compound glycyrrhizin, and rupatadine, and topical application of mupirocin and potassium permanganate from our outpatient services, the patient was then hospitalized with a recurrent condition. He frequently complained of head swelling and pain, accompanied by nausea and vomiting, and the symptoms were not relieved by celecoxib.

Upon clinical examination, his temperature was $38.3^{\circ} \mathrm{C}$; blood pressure, $145 / 88 \mathrm{mmHg}$; heart rate, 125 beats/minute; and respiratory rate, 20 breaths per minute. Physical examination showed many insect bite-like erosions; ruptures; scabs on the face, neck, limbs, and trunk; an orange wheal-like rash; clustered herpes eruptions covering the body; swollen lips; and obvious tenderness. Laboratory values included a white blood cell count of $18,010 / \mu \mathrm{L}$ (normal, 4000-10,000), C-reactive protein $60.9 \mathrm{mg} / \mathrm{L}$ (normal, <8.0), procalcitonin (PCT) $0.062 \mathrm{ng} /$ $\mathrm{mL}$ (normal, 0-0.05), erythrocyte sedimentation rate (ESR) $49 \mathrm{~mm} / \mathrm{h}$ (normal, <21), total $\mathrm{IgE}>1000 \mathrm{KU} / \mathrm{L}$ (normal, <0-60), herpes simplex virus antibody IgG positive, galactomannan (GM) test 5.244 (normal, 0-0.5); other examinations showed no abnormality. After consultation with the infection department, fungal infection was considered, and empirical therapy with liposomal amphotericin B (AMB) $50 \mathrm{mg}$ ivd qd was initiated. Skin tissue and (CSF) were sent for microbiological examination, and skin tissue also underwent pathological examination.

The pathological skin biopsy results showed necrotic and absent epidermis, and diffuse spore-like and internal septation structures were observed around the vessels and adnexa in the superficial and middle dermis. Skin tissue wet mount slides demonstrated numerous leukocyte-like spherical sporangia with multiple internal septation (Figure 1A); Gram staining showed round yeast-like fungal spores of varying sizes with unclear internal structures (Figure 1B); Gomori methenamine silver (GMS) stain revealed multiple round sporangia with an endospore-like symmetrical arrangement or morula (Figure 1C). Few spore-like structures were found in the specimen of CSF with GMS, while the other two dyeing methods were negative (Figure 1D).

The specimens were inoculated on blood agar, Sabouraud's dextrose agar (SDA), and CHROMagar Candida medium plates. After $24 \mathrm{~h}$ of skin tissue culture, large staphylococcal colonies were found on the blood agar plate, while the other two plates were negative. Three days later, pink and creamy colonies were seen on the CHROMagar Candida plate, which resembled colonies of Candida parapsilosis, while the SDA plate was still negative (Figure 2A).

Colony smear showed spherical sporangia in different sizes with characteristic endospore-like mulberry appearance (Figure 2B); Gram staining revealed Gram-positive yeast-like cells without budding or pseudohyphae (Figure 2C). A few days later, tiny colonies appeared on the SDA plate. After 5 days of incubation, the CSF specimen showed growth of only two creamy white, yeast-like colonies on blood agar, which had the same microscopic morphology as skin tissue (Figure 2D). Initial identification of Prototheca wickerhamii for both specimens was performed using the VITEK2 (BioMerieux) automated system and matrix-assisted laser desorption ionizationtime-of-flight mass spectrometry (MALDI-TOF-MS) (BioMerieux) with 99\% confidence. Definitive identification was confirmed via $16 \mathrm{~S}$ rRNA gene sequencing, revealing $100 \%$ homology with $P$. wickerhamii.

In vitro susceptibility tests were carried out using YeastOne Plate (Thermo Fisher Scientific) and were interpreted following CLSI M27-A guidelines. The minimal inhibitory concentration (MIC) values for individual antifungals were AMB $0.25 \mathrm{mg} / \mathrm{L}$, fluconazole (FCA) $>256 \mathrm{mg} / \mathrm{L}, 5$-flucytosine $(5-\mathrm{FC})>64 \mathrm{mg} / \mathrm{L}$, voriconazole (VOR) $1.0 \mathrm{mg} / \mathrm{L}$, itraconazole (ITR) $1.0 \mathrm{mg} / \mathrm{L}$, caspofungin $(\mathrm{CAS})>8 \mathrm{mg} / \mathrm{L}$, anidulafungin $(\mathrm{ANI})>8 \mathrm{mg} / \mathrm{L}$, and posaconazole (POS) $1.0 \mathrm{mg} / \mathrm{L}$.

After the dose of AMB was reduced to $40 \mathrm{mg}$ ivd qd, FCA was added with an initial dose of $200 \mathrm{mg}$, and then, a maintenance dose of $100 \mathrm{mg}$ po was administered for 3 days as the rash improved. AMB was then reduced to $30 \mathrm{mg}$ ivd qd for 3 days due to a progressive elevate in creatinine. The antibiotic regimen was adjusted to AMB $30 \mathrm{mg}$ ivd qd combined with VOR $200 \mathrm{mg}$ po q12 h for 19 days after the identification and drug sensitivity results for $P$. wickerhamii. VOR was then used alone with a first dose of $400 \mathrm{mg}$ ivd $\mathrm{q} 12 \mathrm{~h}$ that was adjusted to a maintenance 

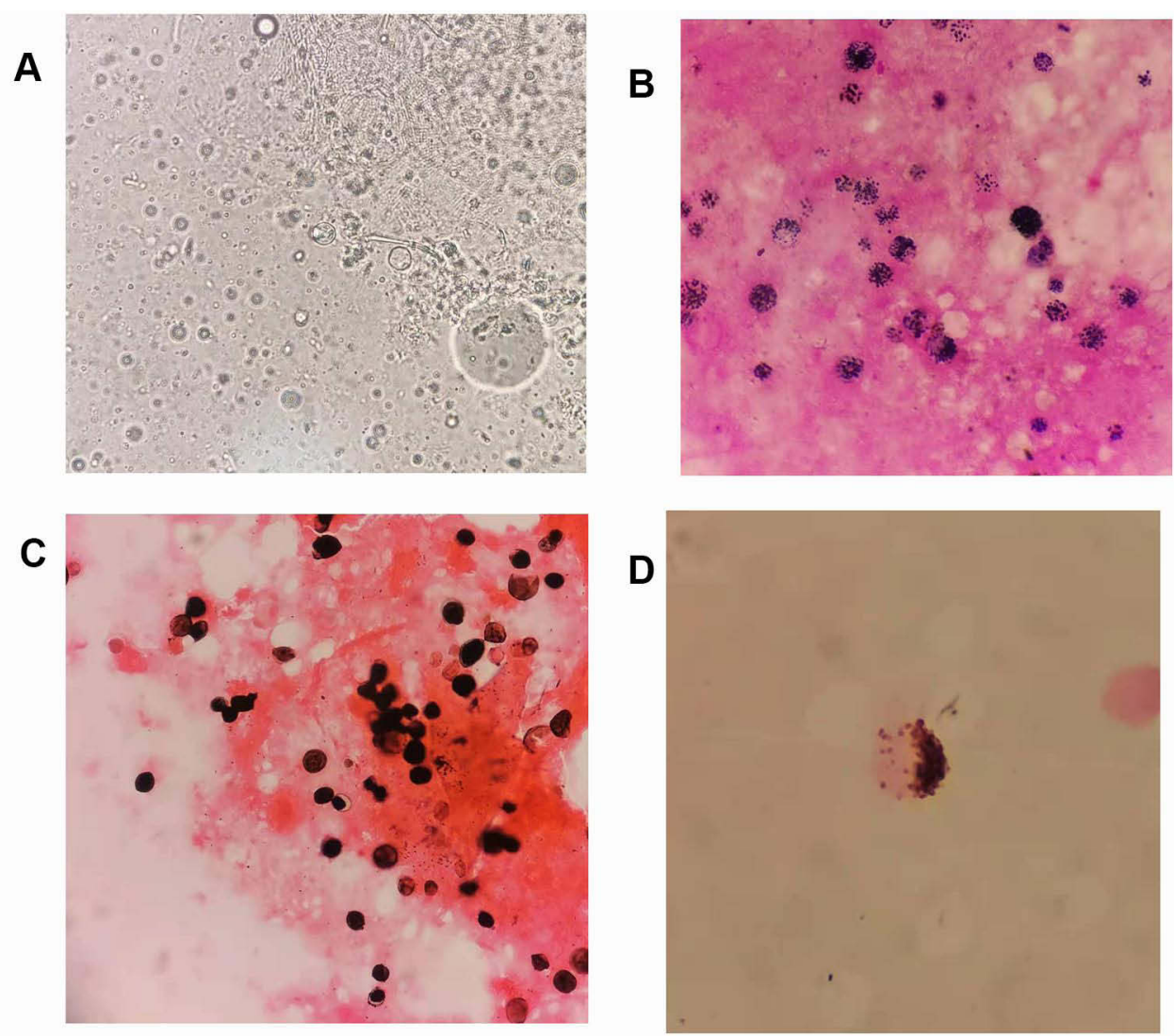

Figure I Direct microscopic morphology of skin tissue and CSF. (A) Direct wet film microscopy of skin tissue, 400×. (B) Gram stain of skin tissue, I000×. (C) GMS of skin tissue, 1000x. (D) GMS of CSF after centrifugation, 1000x.

dose of $250 \mathrm{mg}$ ivd $\mathrm{q} 12 \mathrm{~h}$ for 5 days because of the intractable hypokalemia and gastrointestinal reactions caused by AMB. After two months of treatment, the patient's skin erosion healed, and nervous system symptoms significantly improved. He was discharged but returned to the local hospital for continued treatment.

\section{Discussion}

The biological taxonomy of Prototheca has been controversial, as the genus was first isolated in $1894 .{ }^{4}$ Prototheca spp. are currently classified under the domain of Eukaryota, within the kingdom Viridiplantae, phylum Chlorophyta, class Trebouxiophyceae, order Chlorellales, and family Chlorellaceae. ${ }^{1}$ Presently, there are 8 species of the genus Prototheca including P. wickerhamii, P. zopfii, P. blaschkeae, P. cutis, P. stagnora, P. ulmea, and two novel species named $P$. miyajii sp. nov. and $P$. tumulicola sp. nov. ${ }^{1,5,6}$

When Prototheca is found in human skin, fingernails, respiratory tract, or digestive system, it is usually determined that colonization has occurred. ${ }^{4}$ They can cause rare infections in humans despite their low virulence, and sometimes the infections have fatal outcomes. ${ }^{7}$ Human protothecosis has increased in recent years, with a total of 269 Prototheca infections that has been reported up to March 2021, beginning with the first human case identified on the foot of a Sierra Leone barefoot rice farmer reported by Davies in $1964 .{ }^{8-11} P$. wickerhamii accounts for the majority of Prototheca spp. infections, followed by P. zopfii. ${ }^{12}$

Prototheca spp. infections are usually exogenous and associated with traumatic inoculation from contaminated soil or water. They can also occur either explicitly or implicitly from surgery or catheterization, and even insect bites can cause infections. ${ }^{4}$ Several underlying diseases such as diabetes mellitus, local or systemic steroid use, hematologic malignancy or cancer, AIDS, organ transplantation, alcoholism, peritoneal dialysis, surgery, or catheterization have been associated with the onset of Prototheca infection, especially the use of glucocorticoids. ${ }^{1}$ The patient in this case acquired full-body tattoos eight months before the onset of skin symptoms, which gave us reason 

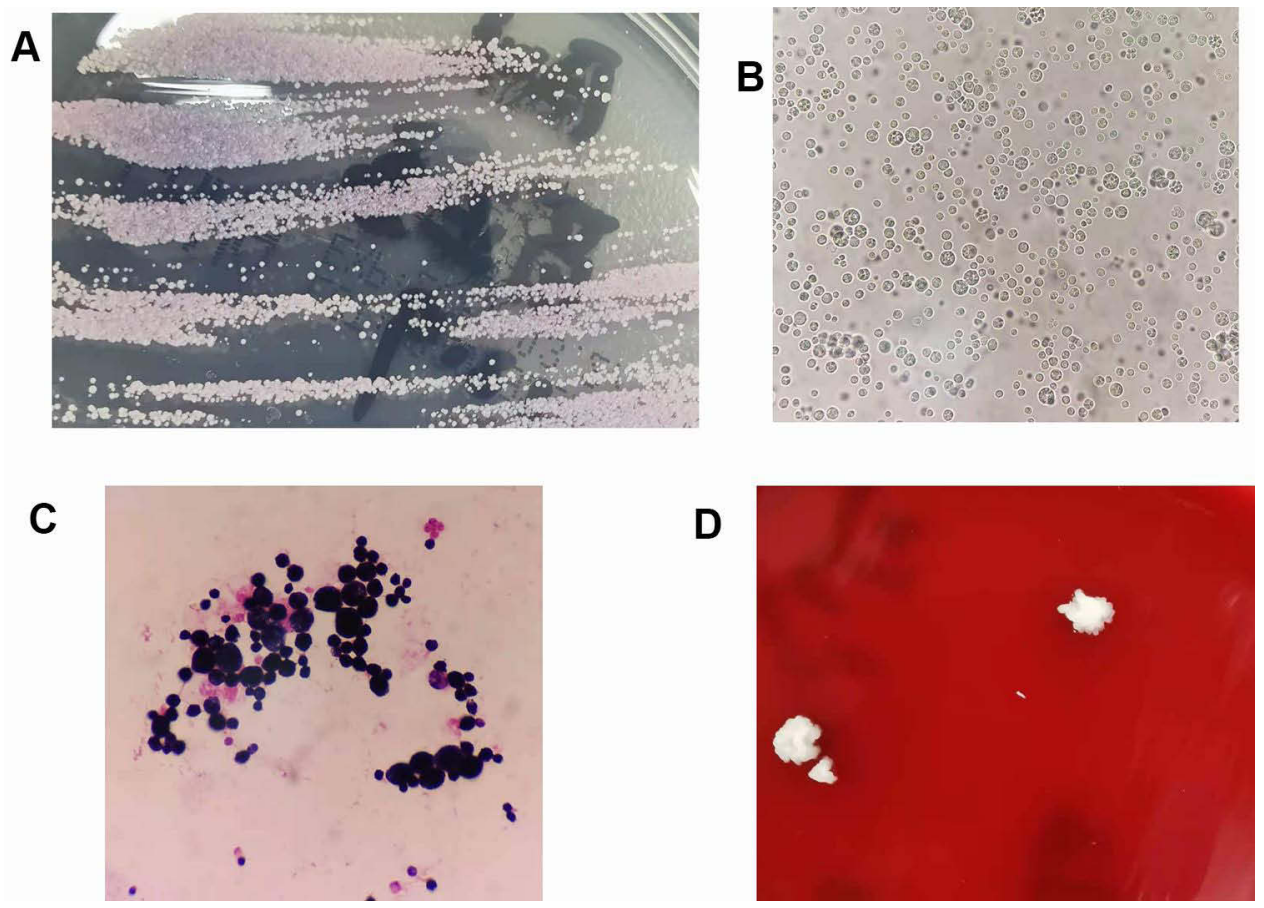

Figure 2 Colonies and microscopic morphology of cultured skin tissue and CSF specimens. (A) Colonies on CHROMagar Candida medium from skin tissue cultures. (B) Wet film microscopy of skin tissue cultures, 40x. (C) Gram stain of skin tissue cultures, 1000×. (D) Colonies on blood agar from CSF cultures.

to suspect that contaminated dye may have been used during the tattooing process, and this was responsible for his infection with Prototheca.

Cutaneous infections, olecranon bursitis, and disseminated infections are the three recognized manifestations of human protothecosis. The skin is the most common human organ that is infected with Prototheca spp. Of 160 cases of human protothecosis, there were $93(58 \%)$ cases that involved a skin infection, as revealed in 20-year followup research reviewed by Todd et al in $2012 .{ }^{13}$ This type of infection usually slowly progresses and has no spontaneous dissolution. ${ }^{1}$ The skin lesions are commonly localized and have a good prognosis with only $1 \%$ mortality. ${ }^{12}$ The infection can occur in both immunocompetent and immunocompromised individuals, but occur with greater frequency in the latter and with a tendency to dissemination. ${ }^{1}$ Infection less commonly occurs in those with olecranon bursitis, which has been reported in patients who had a history of trauma to the elbow or corticosteroid injection. ${ }^{14}$ Disseminated infections usually occur in immunocompromised patients, who have the worst prognosis, with $56 \%$ deaths. ${ }^{12}$ Rare infections occur in those with pre-existing blood infection, urinary tract infection, respiratory tract infection, pneumonia, colpitis, hepatic abscesses, choroiditis, colitis, peritonitis, lymphadenitis, keratitis, endocarditis, fingernail infection, meningitis; additionally, catheter-related infections with Prototheca spp. have been reported. ${ }^{1,10,13,15}$ Khan et al reported the first outbreak of blood Prototheca infections that affected 12 neutropenic patients in a tertiary care chemotherapy oncology unit. $^{2}$

The initial symptom for the patient in our case was a skin infection, but the rash gradually spread throughout the body and progressed to meningitis, although he was immunocompetent. This was a rare case, and there have been only seven previously reported cases of meningitis caused by Prototheca spp., including our case, of which six cases were infected by P. wickerhamii and one case by P. zopfii (Table 1) ${ }^{16-21}$ Among the seven cases of meningitis, there was only one case where confirmation was obtained by the organism being successfully cultured from leptomeningeal tissue, while in the other cases, the organism was cultured in $\mathrm{CSF}^{21}$ The median patient age for these meningitis cases was 17 years, although Prototheca infection tends to occur in the elderly, with a median age of 60 years. ${ }^{12}$ Meningitis caused by the genus has a tendency to occur in young adults, especially minors.

It is also worth noting that only two of seven meningitis patients were immunodeficient, despite disseminated infections commonly occurring in immunocompromised patients. ${ }^{17,18}$ Almost all patients had a history of trauma or 


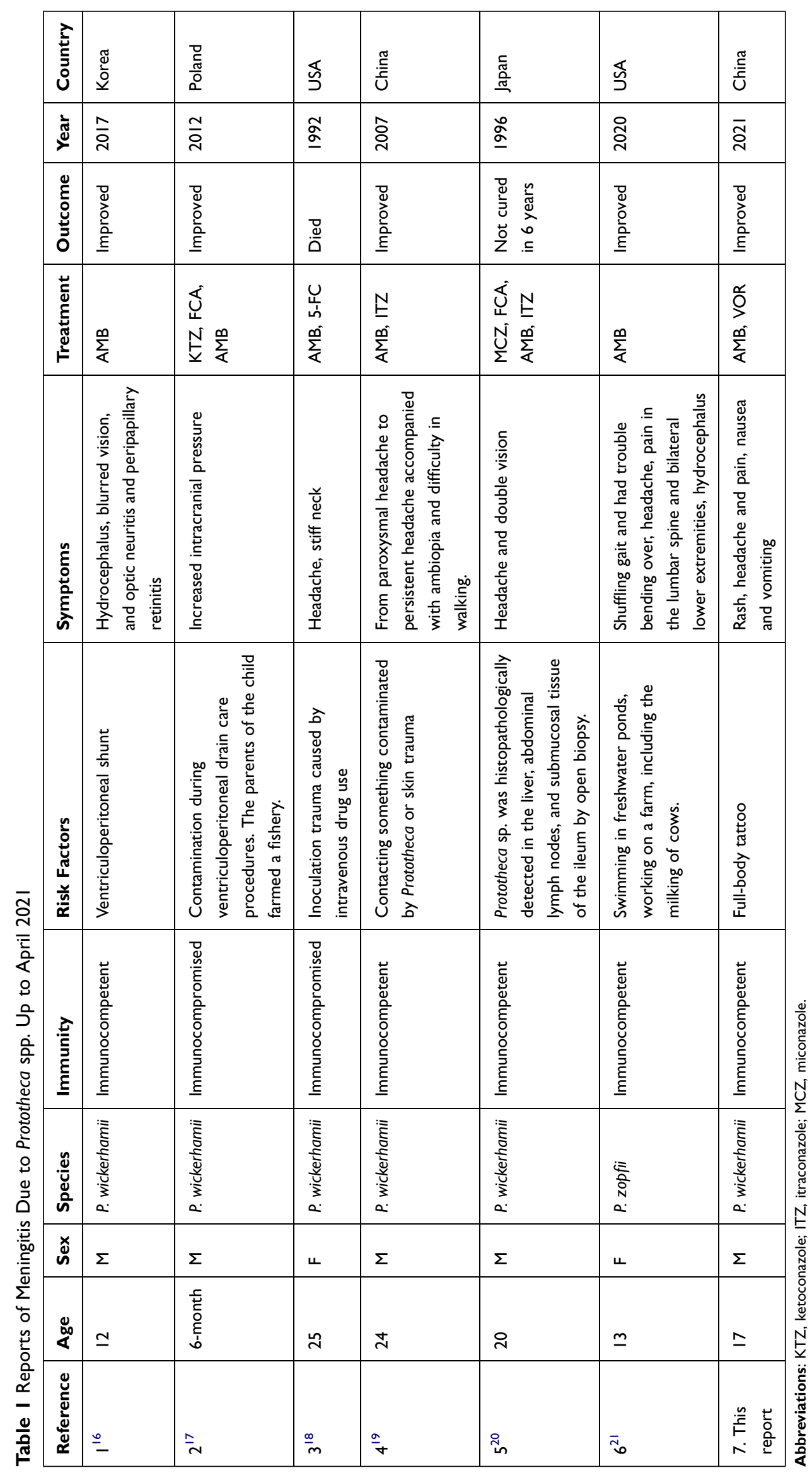


exposure, and headache was the most frequent symptom of meningitis caused by Prototheca. Clinicians should be alert to the possibility of Prototheca meningitis in a young patient who has a history of trauma or exposure and exhibits the symptom of frequent headaches, regardless of whether the patient is immunocompromised or immunocompetent. Chronic process was observed as a feature of Prototheca meningitis, with the infection progressing over months to years. In addition to these seven cases, there were also three brain infections caused by P. zopfii that were previously reported. ${ }^{11,22}$ We did not systematically review these three cases because Prototheca spp. was not confirmed by culture.

Several methods have been used for the identification of Prototheca spp. Microscopic examination is a useful and rapid method for diagnosis but requires clinicians with extensive experience (the microscopic morphology was mentioned above). Histopathological examination reveals the presence of Prototheca, which is a large nonbudding cell with various tissue reactions that mainly include granulomatous inflammation. The genus stains well with GMS and PAS but may be misinterpreted as a non-sporulating cell such as Coccidioides or Cryptococcus neoformans, and a difference in the size of the sporangia may assist in distinguishing them. ${ }^{23}$ Kano et al reported a specific identification of protophycosis that was carried out by an immunohistopathological investigation using rabbit antiserum against $P$. wickerhamii. ${ }^{24}$

The culture of Prototheca spp. is not difficult, and these organisms will readily grow on blood agar. However, some fungal media that include cycloheximide are unsatisfactory for culture. $^{1,2}$ This would explain why there was greater growth of the fungi in our CSF specimen on the blood agar plate as compared to the SDA plate. Unsuccessful isolation of the genus may occur when it is being overgrown by other bacteria and fungi on the culture media. ${ }^{2}$ The Prototheca in our tissue specimen was not identified due to the overgrowth of staphylococcal colonies on the blood agar plate. We suggest the use of a variety of media or a selective media to isolate these fungi when direct microscopic examination is being performed to prevent missed detection in cases of suspected infection. The colony morphology of Prototheca on blood and SDA agar is similar to that of Candida and Cryptococcus, and it is indistinguishable from Candida parapsilosis on CHROMagar Candida medium.

Vitek 2 compact, API 20C AUX, and MALDI-TOF MS in clinical laboratories can rapidly identify the genus to the species level, among which, MALDI-TOF MS can even determine the genotype of $P$. zopfii. ${ }^{4}$ Nucleotide sequencing of $18 \mathrm{~S}$ rDNA and large subunit D1/D2 regions of rDNA has been generally used for the confirmation and classification of Prototheca spp. ${ }^{4}$ Rapid molecular identification of Prototheca based on the ribosomal internal transcribed spacer (ITS) sequence has been also reported. ${ }^{25,26}$ The mitochondrial cytochrome $\mathrm{b}(c y t b)$ gene has been described as a new genetic marker for diagnostics and phylogenetic studies of Prototheca. ${ }^{27}$ The species in our case was confirmed via sequencing of the 16S rRNA gene (which is usually used for the identification of bacteria) and concomitant failure of ITS sequencing, as described above.

There are no specific guidelines or breakpoints for the antimicrobial susceptibility testing (AST) of this organism, and therefore, our case was interpreted following CLSI M27-A guidelines, as were other reported cases. ${ }^{19}$ Among common antifungal agents, AMB, VOR, and ITR usually lowered the MICs as reported. MICs were higher with FCA and 5-FC, and the highest MICs were observed with echinocandin agents such as caspofungin and anidulafungin. ${ }^{1,23}$ It has also been reported that antibacterial agents such as amikacin, tetracycline, and gentamicin often have satisfactory antimicrobial activity in vitro. ${ }^{1,28}$ ASTs are not recommended for routine testing except when empirical therapy has failed, because the AST results are not always equal to the clinical reaction. ${ }^{2}$

There is no standard protocol for the clinical treatment of protophycosis because there are different responses to various treatments. ${ }^{29,30}$ Intravenous AMB is the most widely reported antifungal agent for use in disseminated infections. ${ }^{16-18}$ AMB was given alone or in combination in these seven cases of meningitis and had satisfactory clinical effects except in one case where a patient died. The dose and course of AMB were different in each case, which may be related to the patient's physical condition. In addition, the form of AMB can affect its dose. ${ }^{31}$ Liposomal AMB is a lipid-based formulation that can reduce nephrotoxicity and transfusion-related adverse reactions caused by conventional AMB, but it exerts the same antibacterial effect. ${ }^{32}$ In our case, however, the use of liposomal AMB still resulted in intractable hypokalemia, acute renal impairment, and even gastrointestinal reactions, and thus, it was necessary to reduce the dosage and eventually discontinue the drug. Similar side effects have been reported when using liposomal AMB for the treatment of Prototheca infection, ${ }^{21}$ and this should be clinically noted. Other successful medical treatments such as FCA, ITZ, KTZ, or VOR have been reported. ${ }^{1}$ Surgical operation is considered to be another effective treatment for localized Prototheca infection. 9,33 


\section{Conclusion}

The threat to human health caused by this rare pathogen is increasing. Clinicians should be alert for the possibility of localized infection caused by Prototheca spp. progressing to meningitis, especially when immunocompetent young people undergo traumatic inoculation. Timely diagnosis and treatment are of great significance to the prognosis.

\section{Disclosure}

The authors report no conflicts of interest in this work.

\section{References}

1. Lass-Flörl C, Mayr A. Human protothecosis. Clin Microbiol Rev. 2007;20(2):230-242. doi:10.1128/CMR.00032-06

2. Khan ID, Sahni AK, Sen S, Gupta RM, Basu A. Outbreak of prototheca wickerhamii algaemia and sepsis in a tertiary care chemotherapy oncology unit. Med J Armed Forces India. 2018;74(4):358-364. doi:10.1016/j.mjafi.2017.07.012

3. Min Z, Moser SA, Pappas PG. Prototheca wickerhamii algaemia presenting as cholestatic hepatitis in a patient with systemic lupus erythematosus: a case report and literature review. Med Mycol Case Rep. 2012;2:19-22. doi:10.1016/j.mmcr.2012.12.004

4. Rui K. Emergence of fungal-like organisms: prototheca. Mycopathologia. 2020;185(5):747-754. doi:10.1007/s11046-01900365-4

5. Masuda M, Hirose N, Ishikawa T, Ikawa Y, Nishimura K. Prototheca miyajii sp. nov., isolated from a patient with systemic protothecosis. Int J Syst Evol Microbiol. 2016;66(3):1510-1520. doi:10.1099/ ijsem.0.000911

6. Nagatsuka Y, Kiyuna T, Kigawa R, Sano C, Sugiyama J. Prototheca tumulicola sp. nov., a novel achlorophyllous, yeast-like microalga isolated from the stone chamber interior of the takamatsuzuka tumulus. Mycoscience. 2017;58(1):53-59. doi:10.1016/j.myc.201 6.09 .005

7. Nwanguma V, Cleveland K, Baselski V. Fatal prototheca wickerhamii bloodstream infection in a cardiac allograft recipient. $J$ Clin Microbiol. 2011;49(11):4024-4025. doi:10.1128/JCM.05305-11

8. Davies RR, Spencer H, Wakelin PO. A case of human protothecosis. Trans R Soc Trop Med Hyg. 1964;58(5):448-451. doi:10.1016/00359203(64)90094-X

9. Zhao F, Chen M, Fu Y. Multiple cutaneous infections caused by prototheca wickerhamii. J Clin Lab Anal. 2020;34(11):e23492. doi:10.1002/jcla.23492

10. Minato K, Yoshikawa M, Nakanishi H, Hasegawa K. Long term follow-up of prototheca keratitis: a case report. Int Med Case Rep J. 2020;13:503-506. doi:10.2147/IMCRJ.S268696

11. Herold S, Klodt T, Toelle D, et al. Lethal systemic and brain infection caused by Prototheca zopfii algae in a patient with acute myeloid leukemia. Med Mycol Case Rep. 2021;32(2):17-20. doi:10.1016/j. mmcr.2021.01.004

12. Todd JR, Matsumoto T, Ueno R, et al. Medical phycology 2017. Med Mycol. 2018;56(suppl_1):S188-S204. doi:10.1093/mmy/myx162

13. Todd JR, King JW, Oberle A, et al. Protothecosis: report of a case with 20-year follow-up, and review of previously published cases. Med Mycol. 2012;50(7):673-689. doi:10.3109/1369378 6.2012.677862

14. Yagnik K, Bossé R, Reppucci J, Butts R, Islam S, Cannella AP. Case report: olecranon bursitis due to Prototheca wickerhamii in an immunocompromised patient. Am J Trop Med Hyg. 2019;100(3):703-705. doi:10.4269/ajtmh.18-0818
15. Pascual JS, Balos LL, Baer AN. Disseminated Prototheca wickerhamii infection with arthritis and tenosynovitis. $J$ Rheumatol. 2004;31 (9):1861-1865.

16. Ahn A, Choe YJ, Chang J, et al. Chronic eosinophilic meningoencephalitis by Prototheca wickerhamii in an immunocompetent boy. Pediatr Infect Dis J. 2017;36(7):687-689. doi:10.1097/INF.00000 00000001552

17. Zak I, Jagielski T, Kwiatkowski S, Bielecki J. Prototheca wickerhamii as a cause of neuroinfection in a child with congenital hydrocephalus. First case of human protothecosis in Poland. Diagn Microbiol Infect Dis. 2012;74(2):186-189. doi:10.1016/j.diagmic robio.2012.06.015

18. Kaminski ZC, Kapila R, Sharer LR, Kloser P, Kaufman L. Meningitis due to Prototheca wickerhamii in a patient with AIDS. Clin Infect Dis. 1992;15(4):704-706. doi:10.1093/clind/15.4.704

19. Zhang QQ, Zhu LP, Weng XH, Li L, Wang JJ. Meningitis due to Prototheca wickerhamii: rare case in China. Med Mycol. 2007;45 (1):85-88. doi:10.1080/13693780601003835

20. Takaki K, Okada K, Umeno M, et al. Chronic Prototheca meningitis. Scand J Infect Dis. 1996;28(3):321-323. doi:10.3109/00365549 609027183

21. Joerger T, Sulieman S, Carson VJ, Fox MD. Chronic meningitis due to Prototheca zopfii in an adolescent girl. J Pediatric Infect Dis Soc. 2021;10(3):370-372. doi:10.1093/jpids/piaa049

22. Hench J, Roschanski N, Hewer E, et al. Granulomatous encephalitis: protothecosis excluded? Histopathology. 2016;69(6):1082-1084. doi:10.1111/his. 13020

23. Katwilat P, Chongtrakool P, Muangsomboon S, Jitmuang A. Prototheca wickerhamii prepatellar bursitis in an immunocompetent woman: a case report. J Mycol Med. 2019;29(4):361-364. doi:10.1016/j.mycmed.2019.100901

24. Kano R, Sobukawa H, Suzuki M, et al. Immunohistopathology of Prototheca wickerhamii in cutaneous lesions of protothecosis. Med Mycol J. 2014;55(1):E29-E32. doi:10.3314/mmj.55.E29

25. Hirose N, Nishimura K, Inoue-Sakamoto M, Masuda M. Ribosomal internal transcribed spacer of Prototheca wickerhamii has characteristic structure useful for identification and genotyping. PLoS One. 2013;8(11):e81223. doi:10.1371/journal.pone.0081223

26. Marques S, Huss VA, Pfisterer K, Grosse C, Thompson G. Internal transcribed spacer sequence-based rapid molecular identification of Prototheca zopfii and Prototheca blaschkeae directly from milk of infected cows. J Dairy Sci. 2015;98(5):3001-3009. doi:10.3168/ jds.2014-9271

27. Jagielski T, Gawor J, Bakuła Z, Decewicz P, Maciszewski K, Karnkowska A. cytb as a new genetic marker for differentiation of Prototheca species. J Clin Microbiol. 2018;56(10):e00584-e00518. doi:10.1128/JCM.00584-18

28. Shahan TA, Pore RS. In vitro susceptibility of Prototheca spp. to gentamicin. Antimicrob Agents Chemother. 1991;35(11):2434-2435. doi:10.1128/AAC.35.11.2434

29. Krcméry V Jr. Systemic chlorellosis, an emerging infection in humans caused by algae. Int $J$ Antimicrob Agents. 2000;15 (3):235-237. doi:10.1016/S0924-8579(00)00150-3

30. Wang F, Feng P, Lin Y, et al. Human cutaneous protothecosis: a case report and review of cases from Mainland China, Hong Kong, and Taiwan. Mycopathologia. 2018;183(5):821-828. doi:10.1007/s11046018-0292-3

31. Hamill RJ. Amphotericin B formulations: a comparative review of efficacy and toxicity. Drugs. 2013;73(9):919-934. doi:10.1007/ s40265-013-0069-4

32. Lemke A, Kiderlen AF, Kayser O. Amphotericin B. Appl Microbiol Biotechnol. 2005;68(2):151-162. doi:10.1007/s00253-005-1955-9

33. Telkes G, Dezsö K, Doros A, Mathe Z. Successful treatment of the gastrointestinal manifestation of prototheca in a kidney transplant recipient: a case report. Transplant Proc. 2018;50(10):3928-3931. doi:10.1016/j.transproceed.2018.07.026 


\section{Publish your work in this journal}

Infection and Drug Resistance is an international, peer-reviewed openaccess journal that focuses on the optimal treatment of infection (bacterial, fungal and viral) and the development and institution of preventive strategies to minimize the development and spread of resistance. The journal is specifically concerned with the epidemiology of antibiotic resistance and the mechanisms of resistance development and diffusion in both hospitals and the community. The manuscript management system is completely online and includes a very quick and fair peerreview system, which is all easy to use. Visit http://www.dovepress.com/ testimonials.php to read real quotes from published authors. 